\title{
Costos de bolsillo de pacientes con diagnóstico de tuberculosis en Colombia
}

\author{
Out-of-pocket costs of patients diagnosed with tuberculosis in Colombia
}

\author{
Lina María Martínez-Sánchez ${ }^{1, a}$, Laura Mejía-Cardona ${ }^{1, b}$, Evert Armando Jiménez-Cotes ${ }^{1, b}$, Luis \\ Felipe Álvarez-Hernández ${ }^{1, b}$, Diana Marcela Castrillón-Velilla ${ }^{1, c}$, Gustavo González Palomino, ${ }^{2, d}$, \\ Carlos Lázaro Arroyo ${ }^{2, d}$, Elsa María Doria-Romero ${ }^{2, d}$, Paulina Ayazo-Villadiego ${ }^{2, e}$, Ana Karina Ibañez- \\ Gallego $^{2, e}$, José Mauricio Hernández-Sarmiento ${ }^{1,3, f}$ \\ ${ }^{1}$ Universidad Pontificia Bolivariana, Medellín, Colombia. ${ }^{2}$ Universidad Pontificia Bolivariana, Montería, Colombia. ${ }^{3}$ Corporación para Investigaciones Biológicas ClB, Medellín, \\ Colombia.

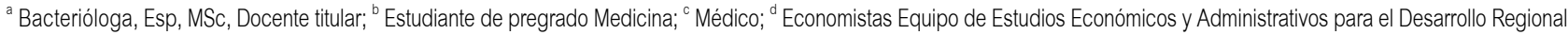 \\ ESDER; ${ }^{e}$ Estudiante de pregrado Economí; ${ }^{\dagger} \mathrm{MD}, \mathrm{MSc}, \operatorname{PhD}(\mathrm{a})$ Docente Asociado.
}

An Fac med. 2017;78(1):37-40/ http://dx.doi.org/10.15381/anales.v78i1.13019

\section{Correspondencia}

Lina María Martínez-Sánchez linam.martinez@upb.edu.co

Calle 78 B N 72 a 109, Medellín,

Colombia.

Recibido, 5 agosto 2016

Evaluado, 6 noviembre 2016

Aceptado, 17 noviembre 2016

Ningún conflicto de interes. Fuente de financiación: Universidad Pontificia Bolivariana.

Citar como: Martínez-Sánchez LM, Mejía-Cardona L, Jiménez-Cotes EA, Álvarez-Hernández LF, CastrillónVelilla DM, González Palomino G, Lázaro Arroyo C, Doria-Romero EM, Ayazo-Villadiego P, IbañezGallego AK, Hernández-Sarmiento JM. Costos de bolsillo de pacientes con diagnóstico de tuberculosis en Colombia. An Fac med. 2017;78(1):37-40. DOI: http://dx.doi. org/10.15381/anales.v78i1.13019
Resumen

Introducción. La tuberculosis (TB) es una enfermedad infecto-contagiosa producida por micobacterias; según datos de la Organización Mundial de la Salud, un tercio de la población mundial está infectada. Para combatirla se ha empleado la estrategia DOTS (Directly Observed Therapy Short Course), efectiva para el diagnóstico, tratamiento y monitoreo de la tuberculosis. Objetivo. Estimar costos de bolsillo que asumen los pacientes con tuberculosis, que reciben tratamiento bajo la estrategia DOTS. Diseño. Estudio observacional descriptivo, prospectivo. Lugar. Tres ciudades de Colombia (Medellín, Montería y Quibdó). Participantes. Pacientes con diagnóstico de TB. Intervenciones. Se utilizó un instrumento de recolección que incluía variables relacionadas con los costos de bolsillo directos e indirectos. El análisis se hizo en el programa SPSS versión 17,0 y STATA 11; a las variables cuantitativas se les estimó media y desviación estándar, mientras que a las cualitativas proporciones. Resultados. Participaron 91 pacientes que se encontraban en tratamiento bajo la estrategia DOTS. El promedio de edad fue $39,3 \pm 20$ años; la mayoría vivía con sus familiares. Los ingresos mensuales de los pacientes tuvieron una media de 422863 COP (1€ $=3149$ COP) y los gastos directos más altos generados por el tratamiento fueron los destinados al desplazamiento y ayudas diagnósticas, con una media de 8181 y 7630 COP, respectivamente. Conclusiones. Los costos asumidos por los pacientes bajo la estrategia DOTS fueron altos, incluso cuando el tratamiento se entrega gratuitamente. La modificación de la estrategia para evitar costos en los pacientes podría impactar disminuyendo el abandono del tratamiento por los mismos.

Palabras clave. Tuberculosis; Costos y Análisis de Costo; Promoción de la Salud; Terapia por Observación Directa.

Abstract

Introduction: Tuberculosis (TB) is a contagious disease caused by mycobacteria. According to the World Health Organization, one third of the world's population is infected. The directly observed therapy short course (DOTS) strategy has been used effective for the diagnosis, treatment and monitoring of tuberculosis. Objective: To estimate the out-of-pocket costs of TB patients who receive treatment under the DOTS strategy. Design: Descriptive prospective and observational study. Setting: Three cities in Colombia (Medellin, Monteria and Quibdo). Participants: Patients diagnosed with TB. Interventions: An instrument was used that included variables related to direct and indirect out-of-pocket costs. The analysis was done using the SPSS version 17.0 and STATA 11; mean and standard deviation were estimated for quantitative variables, and proportions for qualitative variables. Results: The DOTS strategy was applied in 91 patients. The average age was 39.3 \pm 20 years; most patients lived with their families. The monthly income of the patients was 422863 COP (1€ $=3149 \mathrm{COP}$ ) in average and the higher direct costs generated by the treatment were those for traveling and diagnostic aids, with an average cost of 8181 and 7630 COP respectively. Conclusions: The costs assumed by patients under the DOTS strategy were high, even when treatment was provided free of charge. The modification of the strategy to avoid costs in patients could decrease treatment dropout.

Keywords: Tuberculosis; Costs; Cost Analysis; Health Promotion; Directly Observed Therapy. 


\section{INTRODUCCIÓN}

La tuberculosis (TB) es una enfermedad infecto-contagiosa producida por micobacterias, que compromete principalmente los pulmones, pero también puede afectar diferentes órganos y tejidos en el humano. Según datos de la Organización Mundial de la Salud (OMS), un tercio de la población mundial está infectada con $M$. tuberculosis y de estos solo unos pocos desarrollan la enfermedad a lo largo de su vida ${ }^{(1)}$. Aunque los avances tecnológicos han propiciado el mejoramiento de la calidad de vida y un mayor acceso a los servicios de salud, la TB continúa siendo un gran problema de salud pública a nivel global. En 2014, la tuberculosis generó una mortalidad de 1,5 millones de personas, de las cuales 0,4 millones correspondían a personas positivas al virus de inmunodeficiencia humana $(\mathrm{VIH})^{(2)}$. En Colombia se reportan anualmente más de 12000 casos nuevos de TB en todas sus formas, lo que indica que continúa siendo una enfermedad con elevadas tasas de incidencia en nuestro medio ${ }^{(3)}$.

Debido a la alta prevalencia de la TB a lo largo de la historia, se han desarrollado diferentes estrategias de promoción y prevención que buscan su control. La primera fue en 1921 con el desarrollo de la vacuna antituberculosa del bacilo Calmette-Guérin (BCG). La segunda, en 1946, cuando la Unión Internacional Contra la Tuberculosis y la OMS establecieron la estreptomicina como tratamiento para la TB; posteriormente, en 1957, el 'método de Edimburgo', basado en el uso de 3 medicamentos, se convirtió en el estándar de oro antituberculoso. Finalmente en 1950, Karel Styblo desarrolló la estrategia DOTS (por sus siglas en inglés, directly observed therapy short course) o tratamiento acortado directamente supervisado, la cual fue adoptada en 1995 por la OMS como estrategia mundial contra la TB; hoy día es implementada como una estrategia obligatoria. Para el año 2000, 27\% de los casos de TB a nivel mundial habían sido tratados con ella ${ }^{(4-6)}$. Acatando la norma, el Ministerio de la Protección Social de Colombia expidió la resolución 412, que definió el cumplimiento de la estrategia
'Tratamiento Acortado Supervisado con Calidad', DOTS-TASO ${ }^{(3)}$.

DOTS es una estrategia práctica y efectiva para el diagnóstico, tratamiento y monitoreo de la TB. Dicha estrategia incluye ciertos componentes que aportan a la detección de la enfermedad, como el examen directo con microscopio del esputo, quimioterapia, adecuado suplemento de medicamentos, una evaluación sistemática y asequible para todo paciente diagnosticado, entre otros ${ }^{(7)}$.

Es importante que el tratamiento de la TB comience inmediatamente después de ser diagnosticada. Hay factores que contribuyen en el retraso de la recuperación y la generación de multidrogorresistencia (MDR) a los fármacos, como la falta de adherencia al protocolo de tratamiento prescrito, modificaciones inadecuadas a la dieta, tratamiento inapropiado, demoras en la entrega del medicamento, falta de supervisión en la distribución, tiempo y mal uso del tratamiento ${ }^{(8)}$.

El objetivo del presente estudio consistió en estimar los costos de bolsillo que asumen los pacientes con tuberculosis que reciben tratamiento anti TB bajo los lineamientos de la estrategia DOTS.

\section{MÉTODOS}

Se realizó un estudio observacional descriptivo y prospectivo en el que participaron personas con diagnóstico de TB pulmonar y extrapulmonar de las ciudades de Medellín, Montería y Quibdó (Colombia), las cuales cumplieron con los siguientes criterios de selección: edad $\geq$ 18 años, con diagnóstico de TB pulmonar y extrapulmonar que recibían tratamiento bajo la estrategia DOTS al menos dos meses antes, con nivel de lecto-escritura básico o superior y que aceptaron participar mediante la firma del consentimiento informado. La muestra fue a conveniencia, incluyendo pacientes que vivieran en las tres ciudades. Se aplicó un instrumento para la recolección de información que incluyó las variables sociodemográficas y económicas relacionadas con los costos directos e indirectos que constituían todos aquellos implicados con el bolsillo del paciente. Los costos directos se refirieron al gasto monetario que hace el paciente para cubrir necesidades como medicamentos, exámenes, copagos de salud, entre otros; y los costos indirectos fueron todos los que implican un gasto de dinero secundario debido al tiempo invertido en los diferentes procesos que intervienen en la enfermedad, además de la incapacidad laboral, entre otros.

La recolección de información se realizó mediante una entrevista personal en la que se aplicó el instrumento previamente estandarizado para consignar los datos. La información recolectada fue procesada y analizada en los programas SPSS versión 17,0 (SPSS Inc; Chicago, Illinois, EE UU) y STATA 11. A las variables cuantitativas se les estimó la media y la desviación estándar, mientras que a las cualitativas se les calcularon proporciones. La investigación contó con la aprobación del Comité de Ética de la Escuela de Ciencias de la Salud de la Universidad Pontificia Bolivariana. Además, se guardó la confidencialidad de los datos.

\section{RESULTADOS}

Se entrevistó un total de 91 pacientes, 35 en Medellín, 35 en Montería y 21 en Quibdó, durante el período comprendido entre marzo y octubre del año 2013; todos se encontraban en la segunda fase de tratamiento farmacológico bajo la estrategia DOTS en alguna de las instituciones del sistema público de salud de dichas ciudades.

Respecto a las características demográficas, se pudo observar que el promedio de edad fue de $39,3 \pm 20$ años, los pacientes más jóvenes fueron de Quibdó y los mayores de Montería, con una media de edad de 31 y 47 años, respectivamente. La mayoría vivía con sus familiares y convivía con aproximadamente 4 a 5 personas en la casa donde habitaban (ver tabla 1).

Los ingresos mensuales de los pacientes con diagnóstico de tuberculosis que recibían tratamiento bajo la terapia DOTS tenían una media de 422863 COP (DS 307 874), y los gastos directos más altos generados por el tratamiento fueron los destinados al desplazamiento y ayudas 
Tabla 1. Características demográficas de los pacientes encuestados.

\begin{tabular}{cccc} 
Características & \multicolumn{3}{c}{ Ciudad } \\
\cline { 2 - 4 } Media de edad (DE) & Medellín & Montería & Quibdó \\
Sexo (M/F) & $35,5(19,8)$ & $47,3(20,8)$ & $31,7(13,5)$ \\
No. de convivientes & $27 / 8$ & $22 / 13$ & $8 / 13$ \\
\hline
\end{tabular}

Tabla 2. Gastos directos de los pacientes que recibian tratamiento bajo la terapia DOTS.

\begin{tabular}{cc} 
Variable & Media* (DE) \\
Copagos de salud & $2518(14267)$ \\
Gastos de desplazamiento & $8181(20084)$ \\
Gastos de transporte & $3373(10176)$ \\
Gastos en consultas adicionales & $4168(16380)$ \\
Gastos en desplazamientos de consultas adicionales & $1110(5476)$ \\
Gastos en ayudas diagnósticas & $7630(34937)$ \\
\hline
\end{tabular}

*Los valores están en COP $(1 €=3149$ COP $)$. diagnósticas, con una media de 8181 y 7630 COP, respectivamente (tabla 2).

La TB es una enfermedad que afectó la cotidianidad de los pacientes, generando incapacidades laborales y descuento en los ingresos mensuales con una media de 61719 COP (DS 133 015) (ver tabla 3).

La media de los gastos totales por ciudad fueron 86 994, 26394 y 79810 COP para Medellín, Montería y Quibdó, respectivamente. Por ciudad, los gastos más elevados fueron: para Medellín y Quibdó los gastos de transporte, con 11652 COP y 7435 COP, respectivamente; y para Montería, las ayudas diagnósticas, con 7638 COP.

\section{DISCUSIÓN}

Onazi y col. (9) llevaron a cabo un estudio en Nigeria con pacientes que tenían diagnóstico de tuberculosis y fueron tratados bajo la estrategia DOTS, donde la media de edad fue de $37,7 \pm 12,9$ años y el número de convivientes ${ }^{(5,9)}$, lo que coincide con el presente estudio, en el cual la media de edad fue $39,3 \pm 20$ años y el número de convivientes 4 a 5 . Por el contrario, en un trabajo realizado por Shete y col. ${ }^{(10)}$, la media de edad fue 45 años, pero el número de convivientes fue 5 .
La OMS continúa realizando estrategias para logar el control y eliminación de la enfermedad. Pero, los factores socioeconómicos son una barrera importante, ya que afectan económicamente a los pacientes por la pérdida de productividad asociada a las incapacidades que les genera la enfermedad, como se puede apreciar en los datos previos, que aquejó principalmente a personas en edad laboral ${ }^{(11)}$

El gasto de bolsillo más elevado para los pacientes que reciben tratamiento bajo la terapia DOTS en la investigación realizada por Ukwaja y col. ${ }^{(11)}$ en una población nigeriana, fue el correspondiente al transporte, por un valor de 83200 COP, similar a lo observado en el presente estudio en las ciudades de Medellín y Quibdó. Algo parecido se pudo observar en el estudio realizado por Steffen y col. (12), en Brasil, donde los costos directos más altos fueron el trasporte y la medicación no TB, con 33054 COP y 32153 COP, respectivamente.

En algunas oportunidades, los pacientes buscan tratamiento en un promedio de tres instituciones, lo que genera un incremento en el gasto de bolsillo de los pacientes, principalmente para el trans-

Johns y col. ${ }^{(13)}$ desarrollaron una investigación en Indonesia, donde se comparó el costo del tratamiento DOTS frente a otras estrategias en pacientes diagnosticados con tuberculosis, y reportó un costo bajo respecto a otras estrategias diferentes, incluyendo los gastos directos de los pacientes, que oscilaban entre 511615 COP y 1716484 COP, frente a 460151 COP y 2972818 COP de otras terapias. Las cifras del presente estudio son mucho menores en las tres ciudades, lo que ratifica la importancia de la terapia DOTS frente a las demás prácticas para la erradicación de la TB.

Diferentes estudios en el mundo han concluido que la estrategia DOTS es muy importante para lograr el éxito en el tratamiento de los pacientes. Incluso, se ha dicho que es una alternativa costo-efectiva que permite a los programas ahorrar recursos cuando se la implementa y el paciente termina curado, comparándola con el posible fracaso terapéutico de un paciente, que puede generar consecuencias catastróficas en una comunidad al transmitir la infección a su conviviente ${ }^{(14)}$.

Algunos países han decidido hacer modificaciones al DOTS para evitar que los costos que deben asumir los pacientes para recibir el tratamiento suporte y consultas médicas adicionales ${ }^{(9)}$.

Tabla 3. Variables que intervinieron en los costos indirectos de los pacientes que recibían tratamiento bajo la terapia DOTS.

\begin{tabular}{cc} 
Variable & Media (DE) \\
Incapacidades laborales & $1,8(0,39)$ \\
Días de incapacidad & $62,9(88,2)$ \\
Ingresos descontados por incapacidad & $13250(74620)^{*}$ \\
Gastos complementarios a la enfermedad & $15923(77565)^{*}$ \\
\hline
\end{tabular}

*Los valores están en COP $(1 €=3149$ COP). 
pervisado tengan efectos negativos. La alternativa de hacer la supervisión del tratamiento con trabajadores comunitarios, agentes de salud, o la integración de servicios con ONG locales -alternativa que la OMS ha denominado ENGAGE TB-, han demostrado que pueden mejorar positivamente el impacto de la estrategia DOTS. Esto básicamente porque los pacientes no tienen que trasladarse a los centros de salud, si no que en su sitio de residencia o lugar de trabajo reciben la visita de supervisión. Situación que representa indiscutiblemente un ahorro en tiempo y dinero para los pacientes ${ }^{(15)}$.

Un estudio realizado por Jackson y col. ${ }^{(16)}$ en China, uno de los países con mayor incidencia de TB en el mundo, concluyó que un paciente promedio con TB puede llegar a gastar hasta el 55\% de sus ingresos para lograr la curación de la enfermedad. Aunque muchos de los servicios de salud son gratuitos, costos adicionales, tanto médicos como no médicos, hacen que el paciente deba invertir más de la mitad de sus ingresos en el tratamiento de la enfermedad. En el presente estudio, estos gastos representaron menos del 30\% de los ingresos mensuales. Otro estudio realizado en Malawi por Kemp y col. sugiere que entre los costos de bolsillo que deben asumir el paciente y su familia, y el dinero que deja de recibir el enfermo por sus días de incapacidad, superan la media de ingresos de la población ${ }^{(17)}$.

Es posible que el comportamiento que puede tener la enfermedad en población vulnerable esté relacionado con la alta tasa de abandonos que tiene el programa de TB en Colombia, tal como sucede en otras partes del mundo, representando un problema importante de salud pública a nivel mundial ${ }^{(18)}$.

Con relación a las limitaciones del estudio, se encontró poca cantidad de participantes de acuerdo a la muestra total por ciudad, debido al bajo flujo de pacientes los días dispuestos para la recolección de información.

En conclusión, los costos asumidos por los pacientes bajo la estrategia DOTS en las ciudades de Medellín, Montería y Quibdó fueron muy altos. Aunque en la teoría el diagnostico de TB no requiere de copagos, en el sistema actual de salud de Colombia todos los pacientes reportaron este tipo de gastos. La ciudad donde más gastos asumieron estos pacientes fue Medellín. La mayoría de estos copagos tenían que ver con exámenes complementarios que fueron ordenados por los médicos, y que no están cubiertos por el servicio de salud. Es importante que el programa de control de la tuberculosis en las ciudades de Medellín, Montería y Quibdó involucren en el equipo de trabajo a los convivientes del paciente para entender su condición, mejorar el compromiso y adherencia al tratamiento, reduciendo los gastos de bolsillo causados por la enfermedad.

\section{REFERENCIAS BIBLIOGRÁFICAS}

1. Organización Mundial de la Salud. 10 datos sobre la tuberculosis [Internet]. Ginebra: OMS; 2016 [citada 20 de junio de 2016]. Disponible en: http:// www.who.int/features/factfiles/tuberculosis/es/

2. WHO. Global tuberculosis report 2015. Geneva: WHO; 2015

3. Colombia. Ministerio de Salud y Protección Social, INS, OPS. Plan estratégico Colombia libre de tuberculosis 2010-2015: para la expansión y fortalecimiento de la estrategia alto a la TB [Internet]. 3. ed. Bogotá: Ministerio de Salud y Protección Social; 2009 [citada 20 de junio de 2016]. Disponible en:https://www.minsalud.gov.co/sites/rid/Lists/ BibliotecaDigital/RIDE/NS/PP/ET/plan-estrategicocolombia-libre-tb-2010-15.pdf

4. Unión Internacional Contra la Tuberculosis y Enfermedades Respiratorias. La Unión, 90 años de colaboración e innovación. Bianchi (Perú). La Unión. 2010. versión web. Disponible en: http:// www.theunion.org/what-we-do/publications/ general/spanish/90th_broch_Sp_LR.pdf. Ultima consulta 22-1'-2014.

5. Guias de promoción de la salud y prevención de enfermedades en la salud pública. Guía 11: Guía de atención de la tuberculosis pulmonar y extrapulmonar. Programa de Apoyo a la Reforma de Salud - PARS Ministerio de la Protección Social - MPS. Colombia. Mayo 2007

6. Organización Mundial de la Salud. ¿Qué es DOTS? Una guia para la comprensión de la estrategia de control de la tuberculosis recomendada por la OMS conocida como DOTS. OMS/CDS/CPC/ TB/99.270. 1999
7. Woldeyohannes D, Sisay S, Mengistu B, Kassa H Directly observed treatment short-course (DOTS) for treatment of new tuberculosis cases in Somali Regional State, Eastern Ethiopia: ten years retrospective study. BMC Res Notes. 2015 19;8:357. doi: 10.1186/s13104-015-1325-3.

8. Yekrang Sis $H$, Jannati $A$, AsghariJafarabad M, Ebrahimi- kalan M, Tahery A, Koosha A. The effectiveness of family-based DOTS versus professional-family mix DOTS in treating smears positive to tuberculosis. Health Promot Perspect. 2014;4(1):98-106

9. Onazi O, Gidado M, Onazi M, Daniel O, Kuye J, Obasanya $\mathrm{O}$, et al. Estimating the cost of TB and its social impact on TB patients and their households. Public Health Action. 2015;5(2):127-31. doi:10.5588/pha. 15.0002

10. Shete PB, Haguma P, Miller CR, Ochom E, Ayakaka I, Davis JL, et al. Pathways and costs of care for patients with tuberculosis symptoms in rural Uganda. Int J Tuberc Lung Dis. 2015;19(8):912-7. doi: 10.5588/ijtld.14.0166

11. Ukwaja KN, Alobu I, Lgwenyi C, Hopewell PC. The high cost of free tuberculosis services: patient and household costs associated with tuberculosis care in Ebonyi State, Nigeria. PLoS One. 2013;8(8):e73134. doi:10.1371/journal. pone.0073134

12. Steffen R, Menzies D, Oxlade O, Pinto M, de Castro AZ, Monteiro P, et al. Patients' costs and cost-effectiveness of tuberculosis treatment in DOTS and non-DOTS facilities in Rio de Janeiro, Brazil. PLoS One. 2010;5(11):e14014. doi: 10.1371/ journal.pone.0014014

13. Johns B, Probandari A, Mahendradhata Y, Ahmad RA. An analysis of the costs and treatment success of collaborative arrangements among public and private providers for tuberculosis control in Indonesia. Health Policy. 2009;93(2-3):214-24. doi: 10.1016/j.healthpol.2009.08.004.

14. Baltussen R, Floyd K, Dye C. Cost effectiveness analysis of strategies for tuberculosis control in developing countries. BMJ. 2005;331(7529):1364.

15. Prado TN, Wada N, Guidoni LM, Golub JE, Dietze R, Maciel EL. Cost-effectiveness of community health worker versus home-based guardians for directly observed treatment of tuberculosis in Vitória, Espírito Santo State, Brazil. Cad Saude Publica. 2011;27(5):944-52

16. Jackson S, Sleigh AC, Wang GJ, Liu XL. Poverty and the economic effects of TB in rural China. Int $J$ Tuberc Lung Dis. 2006;10(10):1104-10.

17. Kemp JR, Mann G, Simwaka BN, Salaniponi FM, Squire SB. Can Malawi's poor afford free tuberculosis services? Patient and household costs associated with a tuberculosis diagnosis in Lilongwe. Bull World Health Organ. 2007;85(8):580-5.

18. Fleming MF, Krupitsky E, Tsoy M, Zvartau E, Brazhenko N, Jakubowiak W, McCaul ME. Alcohol and drug use disorders, HIV status and drug resistance in a sample of Russian TB patients. Int J Tuberc Lung Dis. 2006;10(5):565-70. 